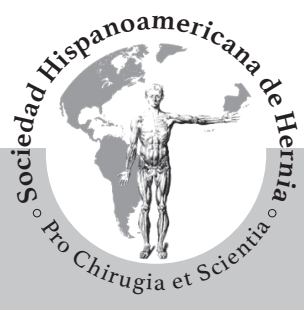

\title{
Variantes del nervio genitofemoral: estudio anatomoclínico para garantizar la seguridad de la triple neurectomía laparoscópica
}

\author{
Variants of genitofemoral nerve: anatomicall study \\ to guarantee the safety of the laparoscopic triple \\ neurectomy
}

\section{Alfredo Moreno-Egea ${ }^{1,2}$, Alfredo Moreno Latorre ${ }^{3}$}

1Jefe. Clínica Hernia. Hospital Universitario La Vega. Murcia (España). ²Profesor de Anatomía Quirúrgica. Departamento de Anatomía Humana. Facultad de Medicina. Universidad Católica San Antonio (UCAM). Murcia (España). ${ }^{3}$ Residente de MFC. Servicio de Urgencias. Hospital Santa Lucía. Cartagena, Murcia (España)

Recibido: 27-04-2018

Aceptado: 21-05-2018

\section{Palabras clave:}

Variantes nerviosas, plexo lumbar, nervio genitofemoral, laparoscopia.

\section{Resumen}

Introducción: El nervio genitofemoral puede ser lesionado en múltiples procedimientos quirúrgicos causando meralgia o inguinodinia. El conocimiento de su anatomía y sus variantes en el espacio retroperitoneal pueden ser esenciales para prevenir su lesión durante un abordaje laparoscópico.

Objetivo: Estudiar el trayecto retroperitoneal del nervio genitofemoral en el cadáver, para comprender y garantizar una cirugía laparoscópica segura.

Método: entre enero de 2014 y mayo de 2017 se disecaron 40 cadáveres adultos formolizados al $10 \%$, en la Cátedra de Anatomía de la Facultad de Medicina de la Universidad Católica de Murcia. El trayecto del nervio genitofemoral fue analizado en base a sus relaciones locales. Todas las disecciones fueron fotografiadas y registradas para posterior valoración.

Resultados: De los 40 plexos lumbares analizados, 32 mostraban un único nervio perforando el músculo psoas (tipo $1,80 \%$ ) y en 8 casos sus dos ramas se dividían en el espesor del músculo emergiendo por separado (tipo 2 , $20 \%$ ). Se describen referencias fiables para localizar los nervios genitofemoral, iliohipogástrico e ilioinguinal en el espacio retroperitoneal.

Conclusión: Los detalles de la anatomía del nervio genitofemoral y sus variantes son importantes para orientar correctamente el diagnóstico de dolor inguinal neural. La formación y experiencia son esenciales para evitar lesiones nerviosas en el abordaje laparoscópico.
Key words:

Nerve variation, lumbar plexus, genitofemoral nerve, laparoscopy.

\section{Abstract}

Introduction: The genitofemoral nerve can be injured in multiple surgical procedures causing meralgia or inguinodynia. Knowledge of its anatomy and variants in the retroperitoneal space can be essential to prevent such injury during a laparoscopic approach.

Objective: To study the retroperitoneal path of the genitofemoral nerve, in autopsy, to understand and guarantee a safe laparoscopic surgery.

Methods: Between January 2014 and May 2017, 40 adult cadavers, 10 \% formalized, were dissected in the Anatomy Department of the Faculty of Medicine of the Catholic University of Murcia. The path of the genitofemoral nerve was analyzed based on its local relationships. All the dissections were photographed and registered for later evaluation.

Results: Of the 40 lumbar plexuses analyzed, 32 showed a single nerve perforating the psoas muscle (type 1,80\%) and in 8 cases its two branches were divided into the muscle thickness emerging separately (Type 2, 20\%). Reliable references are described to locate the genitofemoral, iliohypogastric and ilioinguinal nerves in the retroperitoneal space. Conclusion: The details of the anatomy of the genitofemoral nerve and its variants are important to correctly guide the diagnosis of neural inguinal pain. Training and experience are essential to avoid nerve injuries in the laparoscopic approach.

* Autor para correspondencia: Alfredo Moreno Egea. Clínica Hernia. Hospital Universitario La Vega. C/ Dr. Román Alberca, s/n. 30008 Murcia (España)

Correo electrónico: morenoegeaalfredo@gmail.com

Moreno-Egea A, Moreno Latorre A. Variantes del nervio genitofemoral: estudio anatomoclínico para garantizar la seguridad de la triple neurectomía laparoscópica. Rev Hispanoam Hernia. 2018;6(4):195-200 


\section{Introducción}

Con el advenimiento de las mallas y las técnicas sin tensión, las recidivas han dejado de ser para la mayoría de los cirujanos la complicación más temida de la hernioplastia inguinal ${ }^{1}$. Por el contrario, un mejor seguimiento clínico de los pacientes, una mayor especialización en la pared abdominal y las crecientes exigencias de la sociedad en busca de una mejor calidad de vida han situado al dolor inguinal como la complicación principal de esta cirugía ${ }^{2}$.

Estudios actuales publican una incidencia de dolor inguinal crónico (DIC) que alcanza el 30-54 \% de los pacientes operados, lo que representa un verdadero problema sociosanitario dado el alto porcentaje de población que padece esta patología. Se acepta como DIC a aquel que aparece tras la operación y persiste más de 3 meses, sin existencia de dolor previo. A los 6 meses se debería considerar la reevaluación por un especialista y al año, posiblemente, se podría indicar una neurectomía si no ha respondido a las medidas de tratamiento conservador ${ }^{1-4}$.

La operación de sección neural requiere de unos conocimientos anatómicos precisos. La anatomía regional debe ser la base de una cirugía segura, pero los cirujanos, hasta hoy día, carecen de experiencia y formación en este tema, pues nunca se ha contemplado ni en programas ni en cursos de la especialidad. El acceso a una formación directa sobre cadáveres es imposible para muchos compañeros y los textos clásicos de anatomía no contemplan detalles suficientes sobre el trayecto retroperitoneal ni referencias adecuadas para poder realizar una identificación segura de los nervios del plexo lumbar durante un abordaje laparoscópico ${ }^{5-8}$.

El objetivo de este estudio es describir el trayecto del nervio genitofemoral (GF) en el espacio retroperitoneal, y relacionarlo con los hallazgos de la cirugía laparoscópica, para definir referencias anatómicas útiles para su identificación quirúrgica.

\section{Material y métodos}

En el período de enero de 2014 a enero de 2018, se disecaron 40 cadáveres humanos adultos formolizados al $10 \%, 80$ plexos lumbares como material de estudio final, en la Cátedra de Anatomía de la Facultad de Medicina de la Universidad Católica de Murcia (UCAM). Los cadáveres no presentaban anomalías espinales, tumores regionales ni historia de cirugía o deformidad ortopédica.

Se realizaron disecciones completas del plexo lumbar, tanto de su trayecto retroperitoneal como del intermuscular (abordaje anterior), para facilitar la identificación de los nervios GF, iliohipogástrico (Ih) e ilioinguinal (Ii), en relación con el subcostal (espinal XII) y femorocutáneo. También se disecaron en detalle los músculos psoas (MP), ilíaco (MI), cuadrado lumbar (MCL) y transverso del abdomen (MTA), y sus respectivas aponeurosis y/o fascias (lámina anterior de la fascia toracolumbar y aponeurosis lumboilíaca). Los parámetros observados del nervio GF fueron: formación del plexo lumbar, curso o trayecto retroperitoneal, relación con el músculo psoas y formación de sus ramas.

Las preparaciones fueron registradas fotográficamente para su posterior análisis por un segundo observador. Los datos fueron recogidos de forma prospectiva y analizados después de forma descriptiva. Este estudio representa la continuación de una experiencia previa centrada en los nervios Ih e Ii, ya publicados ${ }^{9-11}$. Los datos fueron comparados con los publicados por otros autores en la literatura.

\section{Anatomía quirúrgica}

El nervio GF (o lumboabdominal de Bichat o Crural de Meckel) se origina de la segunda raíz del plexo lumbar (L2). Atraviesa el MP mayor por su cara anterior a nivel de la tercera vértebra lumbar, discurre hacia abajo sobre su cara anterior envuelto en su aponeurosis, a lo largo del borde externo de las arterias ilíacas primitivas y vasos ilíacos externos (al que da una rama vasomotora). Se sitúa delante de las arterias ilíaca común e ilíaca externa, después de haber cruzado la cara posterior del uréter y termina por encima del ligamento inguinal. Se divide en dos ramas a una altura variable antes de llegar al arco femoral.

- Ramo genital (rG) (o rama espermática externa de Schmidt): penetra en el anillo inguinal profundo del conducto inguinal. Da filetes al MTA y al oblicuo interno del abdomen, hasta dentro del músculo cremáster. Recorre el conducto inguinal a lo largo del ligamento inguinal con el cordón espermático (o ligamento redondo en la mujer) y emerge por el anillo inguinal superficial, para terminar en la piel del escroto, en el hombre, y en los labios mayores, en la mujer.

- Ramo femoral (rF) (o lumbo-inguinal de Schmidt): atraviesa el anillo femoral, lateral a los vasos femorales, para situarse delante de la arteria femoral en el triángulo femoral, y después de 2 o $3 \mathrm{~cm}$ perfora la fascia cribiforme y termina a nivel de la parte anterior y superior del muslo.

\section{Resultados}

El presente estudio fue realizado sobre 40 cadáveres humanos, 8 femeninos y 32 masculinos, exponiendo finalmente 80 piezas del plexo lumbar completo. Las disecciones sobre cadáver permitieron verificar los nervios del plexo lumbar en su totalidad y sus relaciones musculares. En todos los casos se encontraron los nervios Ih y GF, no así el nervio Ii que estaba ausente en 2 plexos $(2.5 \%)$. El nervio GF a nivel retroperitoneal se muestra como un único tronco común, desde su salida sobre la superficie anterior del MP en 32 de los casos (80\%), lo que denominamos como tipo 1 (figs. 1 y 2). Frente a esta variedad más común, en un $20 \%$ de los casos, el nervio GF emerge ya dividido sobre el MP en sus dos ramos. Esta forma de presentación la llamamos tipo 2 (figs. 3 y 4 ).

Anatómicamente, este nervio se identifica bien en el espacio retroperitoneal apareciendo sobre la cara anterior del MP, aunque en 1 caso apareció de forma lateral al mismo (1.25\%). Suele aparecer a unos 4-5 cm de la inserción del músculo y tiene un trayecto de unos 6-7 cm, de forma lineal apareciendo sobre la cara anterior del músculo psoas cubierto siempre por la débil fascia ilíaca, cuya disección es necesaria para poder individualizarlo, lo que supone una referencia básica. A unos $2-3 \mathrm{~cm}$ de la pared posterior se suele dividir en dos ramas, la $\mathrm{rF}$ que entra casi a $90^{\circ}$ respecto al plano muscular por debajo de la cintilla de Thomson sobre la vena ilíaca para emerger sobre la arteria una vez pasado el ligamento inguinal, y la rG que se dirige al borde posterolateral del orificio profundo acompañando a los vasos espermáticos, con notable variabilidad local. Las relaciones son las siguientes: inferiormente con el tendón del psoas, lateral externo con el nervio femoral (a un plano algo más profundo), superior con el uréter y medial con los vasos ilíacos externos. 


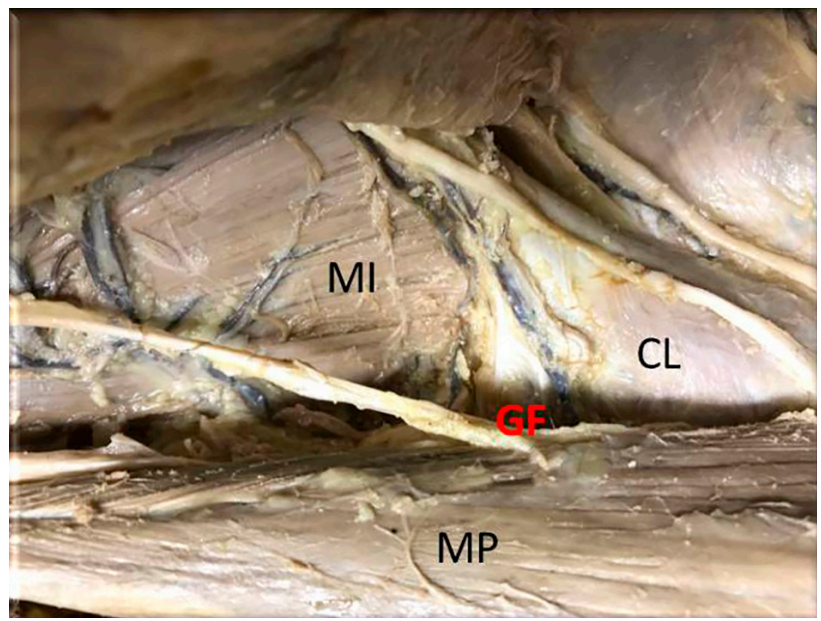

Figura 1. Nervio genitofemoral tipo 1: imagen anatómica (MI: músculo ilíaco; MP: músculo psoas; MCL: músculo cuadrado lumbar; GF: nervio genitofemoral).

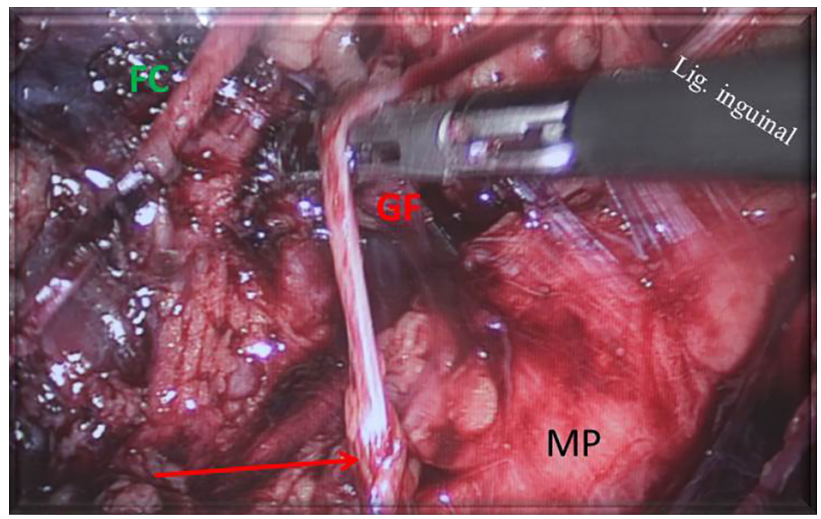

Figura 2. Nervio genitofemoral tipo 1: correlación clínica en una cirugía con abordaje laparoscópico (MP: músculo psoas; GF: nervio genitofemoral; FC: nervio femorocutáneo lateral).

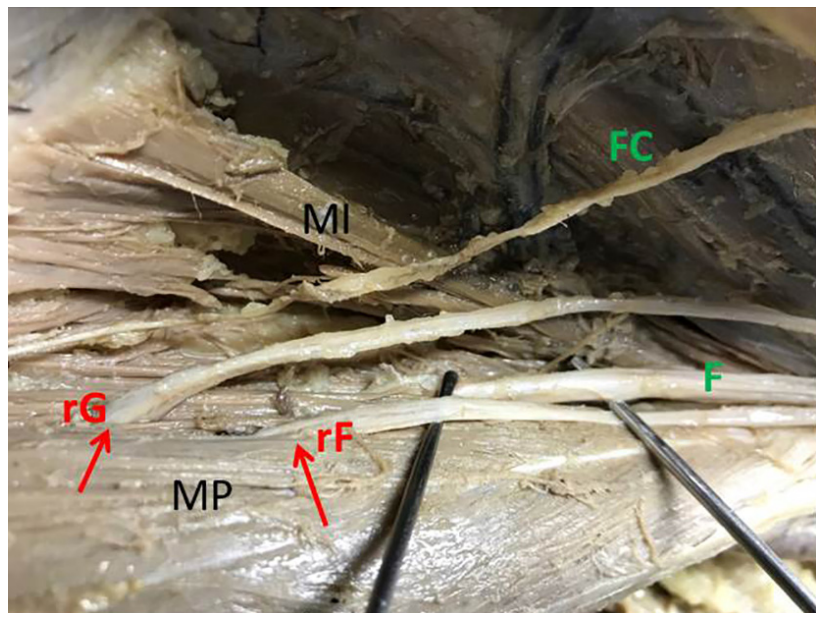

Figura 3. Nervio genitofemoral tipo 2: imagen anatómica (MI: músculo ilíaco; MP: músculo psoas; rG: rama genital del genitofemoral; rF: rama femoral del genitofemoral; FC: nervio femorocutáneo; F: nervio femoral).

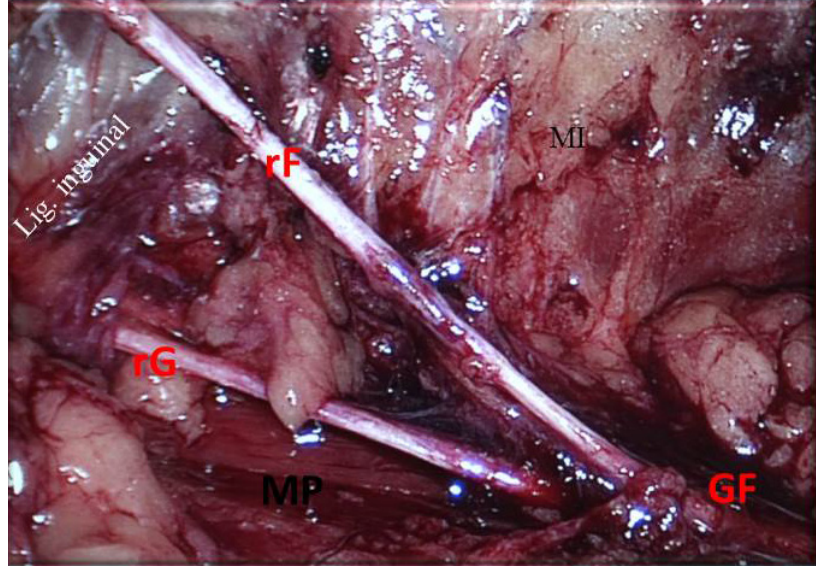

Figura 4. Nervio genitofemoral tipo 2: correlación clínica en una cirugía con abordaje laparoscópico (MI: músculo ilíaco; MP: músculo psoas; rG: rama genital del genitofemoral; $\mathrm{rF}$ : rama femoral del genitofemoral).

En el tipo 1, antes de su bifurcación, podemos definir una zona triangular donde se puede localizar con seguridad el nervio GF y a la que denominamos Triángulo Neuromuscular (fig. 5). Del estudio topográfico regional obtenemos un grupo de referencias que señalamos en la tabla I.

Con respecto al MP, el nervio Ih e Ii aparecen lateralmente, para situarse sobre el MCL y después en el MTA al que perforan a 1-1.5 $\mathrm{cm}$ de la cresta ilíaca posterosuperior formando una referencia que denominamos Cabestrillo Muscular (fig. 6). El nervio FC aparece también lateral al MP pero se sitúa sobre el MI, y el nervio obturador emerge por su borde medial dentro de la pelvis lateral.

\section{Revisión de la literatura}

En la tabla II se recogen los datos analizados en la literatura en cuanto a la tasa de incidencia de variantes en el curso del nervio GF. Arora publica la ausencia del nervio Ih en el $13.3 \%$, del Ii en el $14.9 \%$ y del GF en el $11.6 \%$ de los plexos lumbares. Gindha, ha descrito la ausencia del nervio en el $11.6 \%$ de los casos, y en cuanto a su origen, aunque lo más frecuente es la procedencia de L1-2 (68.3\%), puede venir de T12-L1 (3.3\%), de L1-2-3 (5\%), de L2 (6.6 \%) o de L2-3 (3.3\%) ${ }^{12,13}$. El estudio de Mathias establece que el GF perfora el poas y se divide después de $7 \pm 3.5 \mathrm{~cm}$ en sus dos ramas, en un $58 \%$ (en el $42 \%$ las dos ramas aparecen separadas directamente sobre el músculo) ${ }^{14}$. Para Bergman, esta disposición alcanza el $80 \%$ para un tronco único frente al $20 \%$ como nervio doble directo ${ }^{15}$.

\section{Discusión}

El nervio GF tiene un doble trayecto, retroperitoneal e intraparietal. A diferencia del trayecto intraparietal que es bien conocido por cirujanos y anestesistas, profesionales especializados en la cirugía de las hernias, el recorrido retroperitoneal ha sido poco estudiado y sus referencias anatómicas son escasas e imprecisas para garantizar un abordaje laparoscópico seguro ${ }^{5-8}$. 


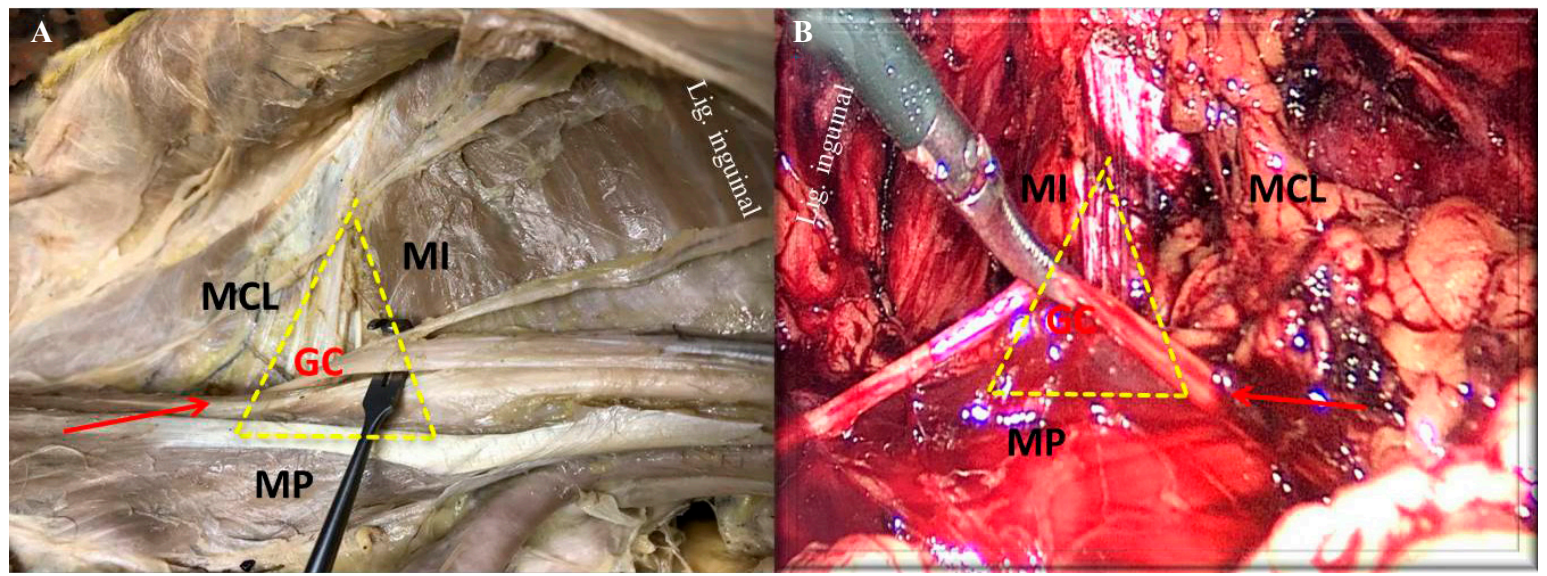

Figura 5. Triángulo neuromuscular como referencia del nervio genitofemoral. A. Imagen anatómica. B. Imagen quirúrgica (MT: músculo transverso abdominal; MI: músculo ilíaco; MP: músculo psoas; GC: nervio genitofemoral o crural).

\begin{tabular}{lll}
\multicolumn{1}{c}{ Referencia } & \multicolumn{1}{c}{ Tabla I. Referencias anatómicas del nervio genitofemoral } & GF \\
\hline Origen & Lateral al psoas & Intramuscular \\
Trayecto & Oblicuo y superoexterno & Vertical y paralelo \\
& Superficie anterior MCL & Superficie anterior MP \\
Fascia (de cubierta) & Lámina anterior & Fascia ilíaca \\
Punto-guía (cirugía) & Fascia toracolumbar & Aponeurosis lumboilíaca \\
Relaciones & Cabestrillo muscular & Triángulo neuromuscular \\
\hline
\end{tabular}

Ih-li: nervio iliohipogástrico e ilioinguinal; GF: nervio genitofemoral; MCL: músculo cuadrado lumbar; MP: músculo psoas; MTA: músculo transverso abdominal.
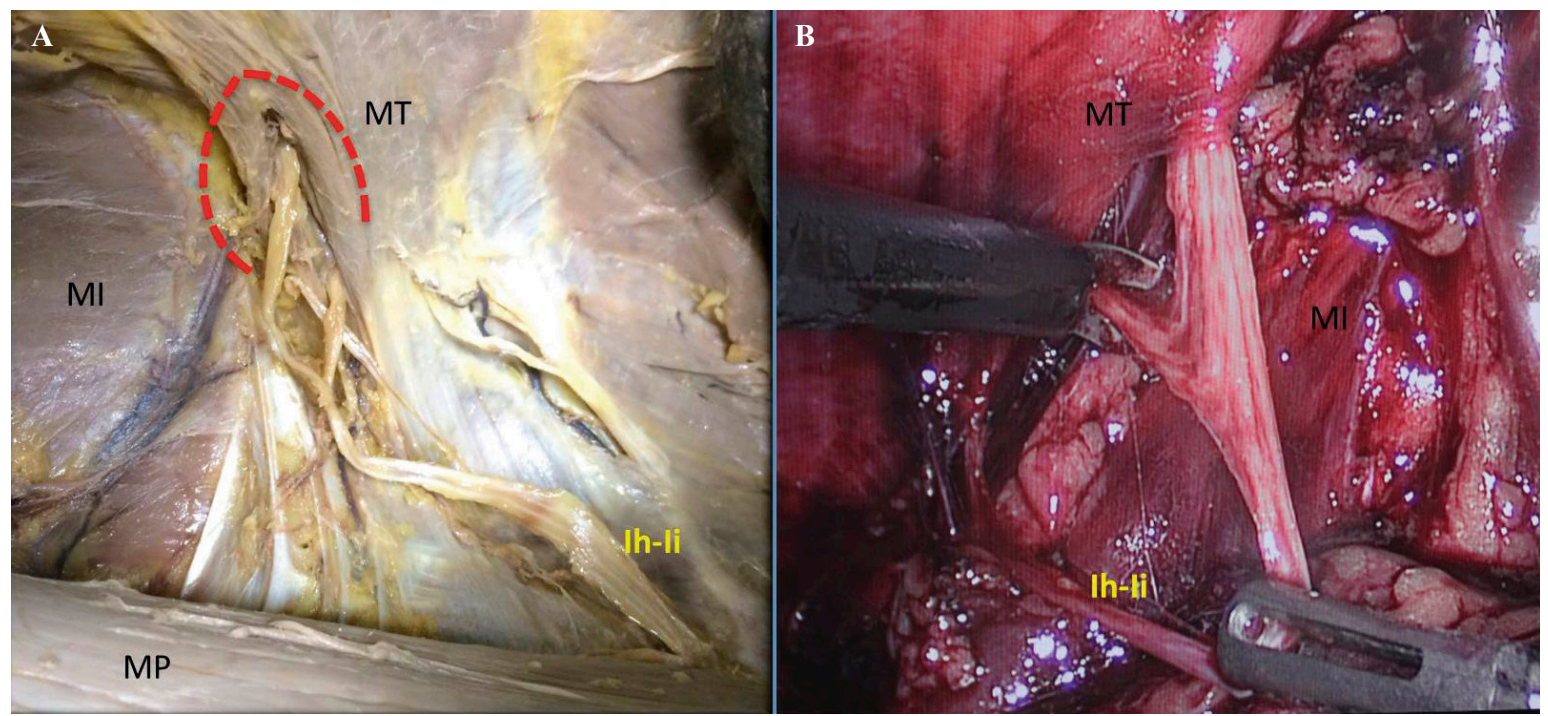

Figura 6. Cabestrillo muscular del transverso del abdomen como referencia del nervio iliohipogástrico e ilioinguinal. A. Imagen anatómica. B. Imagen quirúrgica (MT: músculo transverso abdominal; MI: músculo ilíaco; MP: músculo psoas; Ih-Ii: nervios iliohipogástrico e ilioinguinal). 


\begin{tabular}{llllll}
\multicolumn{6}{c}{ Tabla II. Variantes del nervio genitofemoral: } \\
estudio de la literatura \\
\multicolumn{1}{c}{ Autor } & $\mathrm{n}$ & Tipo 1 & Tipo 2 & No & L1-2 \\
\hline Sim & 60 & 58 & 8,3 & & \\
Mahajan & 60 & 80 & 20 & - & \\
Anloague & 34 & 73,5 & 26,5 & - & \\
Matthias & 32 & 58 & 42 & - & \\
Gindha & 60 & - & - & 11,6 & 68,3 \\
Arora & 60 & - & - & 11,6 & 75 \\
Moreno-Egea & 80 & 80 & 20 & - & 70 \\
\hline
\end{tabular}

n: número de plexos lumbares analizados; tipo 1: tronco único emergiendo del músculo psoas; tipo 2: dos ramas independientes desde el espesor del músculo psoas; no: ausencia del nervio; L1-2: origen en las raíces lumbar 1 y 2 , resto del porcentaje como variantes.

El reconocimiento de variantes o diferencias anatómicas no es solo una curiosidad, es algo esencial para quienes operan, diagnostican o interpretan imágenes de pacientes que padecen problemas reales. Los cirujanos necesitamos extrapolar los síntomas y signos de un posible diagnóstico clínico a unos hechos anatómicos concretos. Por ello las variantes de un nervio son tan importantes a la hora de realizar una operación segura. La incapacidad para diferenciar el nervio GF del Ii o del FC puede conllevar una innecesaria morbilidad. Los nervios del plexo lumbar en general y el GF en particular, se pueden lesionar en cirugías como la hernioplastia inguinal, apendicectomía, cesárea (incisión de Pfannenstiel), histerectomía o vasectomía, nefrectomías o cirugía sobre uréter y próstata, en cirugías de injerto óseo sobre la cresta ilíaca o de la columna (entre un 2 y un $7 \%$ ), en traumatismos abdominales, en la inserción de trócares en laparoscopia o en cirugía laparoscópica retroperitoneal ${ }^{16-20}$

El nervio GF se origina habitualmente de las raíces lumbares L1-2 y aparece en el espacio retroperitoneal perforando la cara anterior del músculo psoas a unos $4.5 \mathrm{~cm}$ de su origen, o sobre su borde lateral en un $20 \%$ de las veces. Puede originarse solo de L2 en un $6.7 \%$ de los casos. En su forma más habitual o tipo 1 desciende como un único nervio sobre la superficie anterior (bajo la fascia ilíaca) unos $7 \mathrm{~cm}$ hasta que se bifurca en 2 ramas antes de alcanzar el ligamento inguinal. Menos frecuentemente, en el tipo 2, el nervio se divide en dos ramas en el espesor del MP y aparecen por separado, la rG primero y después la $\mathrm{rF}$ (tabla I). Otras variantes de los nervios del plexo lumbar referidas en la literatura son: ausencia de Ii, el cual se compensa con la rG del GF que es más gruesa; ausencia de la rG que se sustituye por el Ii, ausencia de la rF que se compensa con el FC lateral, rama del Ii al $\mathrm{rG}$ entrando en el conducto inguinal de forma variable o ausencia de ambas ramas. Estas variaciones neurales son debidas a errores en el desarrollo embriológico y suelen asociarse a anomalías como la hernia inguinal congénita o la criptorquidia ${ }^{21-26}$.

Las frecuentes variantes del nervio GF incrementan la incidencia de lesiones durante la laparoscopia retroperitoneal, sobre todo cuando estamos ante un tipo 2 no reconocido. Entonces se puede manifestar una clínica de neuralgia, meralgia parestésica, inguinodinia, dolor testicular o abolición del reflejo cremastérico, lo que se traduce en un ligero descenso del testículo del lado afecto. Benes, en 2000 ha sugerido llamar a esta entidad síndrome de dolor abdominoinguinal ${ }^{27}$. Por ello, una correcta formación en anatomía regional y de sus variantes es esencial en la prevención de lesiones nerviosas del plexo lumbar, principalmente cuando optamos por el abordaje laparoscópico ${ }^{26-30}$.

En conclusión: a) el dolor inguinal es actualmente de gran interés para el cirujano especializado en pared abdominal; b) el conocimiento y los detalles de la anatomía del nervio GF y sus posibles variantes son importantes para orientar correctamente un diagnóstico de dolor neural; y c) la formación, experiencia y habilidad técnica son esenciales para evitar lesiones nerviosas en el abordaje laparoscópico.

\section{Bibliografía}

1. Loos MJ, Roumen RM, Scheltinga MR. Classifying post-herniorrhaphy pain syndromes following elective inguinal hernia repair. World J Surg. 2007;31:1760-5.

2. Ducic I, West J, Maxted W. Management of chronic postoperative groin pain. Ann Plast Surg. 2008;60:294-8.

3. Lee CH, Dellon AL. Surgical management of groin pain of neural origin. J Am Coll Surg. 2000;191:137-42.

4. Poobalan AS, Bruce J, Smith WC, King PM, Krukowski ZH, Chambers WA. A review of chronic pain after inguinal herniorrhaphy. Clin J Pain. 2003;19(1):48-54.

5. Williams PL, Bannister LH, Berry MM, Collins P, Dyson M, Dussek JE, et al., editors. Gray's Anatomy - The anatomical basis of medicine and surgery. New York: Churchill Livingston; 1995. pp. 378-81.

6. Gilroy A, MacPherson B, Ross L. Prometheus Atlas de Anatomía. Madrid: Ed. Médica Panamericana; 2010. pp. 424-9.

7. Testut L, Latarjet A. Tratado de Anatomía Humana. Madrid: Ed. Salvat (Tomo III); 1982. pp. 307-20.

8. Sobotta J. Atlas de Anatomía Humana. Buenos Aires: Ed. Médica Panamericana. 21. ${ }^{\text {a }}$ ed.; 2000. pp. 274.

9. Moreno-Egea A. Anatomía aplicada a la neurectomía del iliohipogástrico laparoscópica extraperitoneal. Revista Argentina Anatomía Online. 2016;7(1):20-4.

10. Moreno-Egea A. Bases anatómicas para la neurectomía selectiva laparoscópica del nervio ilioinguinal. Rev Hispanoam Hernia. 2016;04:51-8.

11. Moreno-Egea A. Surgical management of postoperative chronic inguinodynia by laparoscopic transabdominal preperitoneal approach. Surg Endosc. 2016;30(12):5222-7.

12. Arora D, Trehan SS, Kaushal S, Chhabra U. Morphology of lumbar plexus and its clinical significance. Int J Anat Res. 2016;4(1): 2007-14.

13. Gindha GS, Arora D, Kaushal S, Chhabra U. Variations in origin of the genitofemoral nerve from the lumbar plexuses in North Indian Population (A cadaveric study). MOJ Anat Physiol. 2015;1(3):00015.

14. Mathias R, Ebmer J, Delon L. Anatomic variability of the ilioinguinal vs genitofemoral the nerve: implications for treatment of groin pain. Plast Reconstr Surg. 2001;108:1618-23.

15. Bergman RA, Afifi AK, Miyauchi R. Genitofemoral nerve. Available from: http://www.anatomyatlases.org/AnatomicVariants/NervousSystem/Text/GenitofemoralNerve.shtml

16. Aasvang E, Kehlet H. Surgical management of chronic pain after inguinal hernia repair. Br J Surg. 2005;92(7):795-801.

17. Irvin W, Andersen W, Taylor P, Rice L. Minimizing the risk of neurologic injury in gynecologic surgery. Obstet Gynecol. 2004;103:374-382.

18. Murovic JA, Kim DH, Tiel RL, Kline DG. Surgical management of genitofemoral neuralgias at the Louisiana State University Health Sciences Center. Neurosurgery. 2005;56:298-303. 
19. O'Brien MD. Genitofemoral neuropathy. Br Med J. 1979;1:1052.

20. Starling JR, Harms BA, Schroeder ME, Eichman PL. Diagnosis and treatment of genitofemoral and ilioinguinal entrapment neuralgia. Surgery. 1987;102:581-6.

21. Geh N, Schultz M, Yang L, Zeller J. Retroperitoneal course of iliohypogastric, ilioinguinal, and genitofemoral nerves: A study to improve identification and excision during triple neurectomy. Clin Anat. 2015;28(7):903-9.

22. Maldonado PA, Slocum PD, Chin K, Corton MM. Anatomic relationships of psoas muscle: clinical applications to psoas hitch ureteral reimplantation. Am J Obstet Gynecol. 2014;211(5):563.e1-6.

23. Mahajan R, Paul S, Mahajan R Jr. Variants of genitofemoral nerve: an insight into its clinical implications. Int J Clin Surg Adv. 2014;2(2):19-28.

24. Shanthanna H. Successful treatment of genitofemoral neuralgia using ultrasound guided injection: a case report and short review of literature. Case Rep Anesthesiol. 2014;2014:371703.
25. Anloague PA, Huijbregts P. Anatomical Variations of the Lumbar Plexus: A Descriptive Anatomy Study with Proposed Clinical Implications. J Man Manip Ther. 2009;17(4):107-14.

26. Magee RK. Genitofemoral Causalgia: A New Syndrome. Can Med Assoc J. 1942;46(4):326-9.

27. Benes J, Nádvornik P, Dolezel J. Abdominoinguinal pain syndrome treated by centrocentral anastomosis. Acta Neurochir (Wien). 2000;142(8):887-91.

28. Lee TK, Yazdi JS, Floro KE, Arenos PT, Lee JR. Protection of the genitofemoral nerve using endoscopic assistance in minimally invasive lateral lumbar fusion. Interdisciplinary Neurosurg Adv Tech and Case Management. 2017;8:4-7.

29. Cesmebasi A, Yadav A, Gielecki J, Tubbs RS, Loukas M. Genitofemoral neuralgia: a review. Clin Anat. 2015;28(1):128-35.

30. Uzmansel D, Aktekin M, Kara A. Multiple variations of the nerves arising from the lumbar plexus. Neurosurgery 2008;5:37-39. 